\title{
Influence of prior 810-nm-diode intracanal laser irradiation on hydrophilic resin-based sealer obturation
}

Cacio Moura-Netto(a)

Renato Miotto Palo(b)

Samira Esteves Afonso Camargo(c) Carol Jent ${ }^{(d)}$

Renato de Toledo Leonardo(e)

Marcia Martins Marques ${ }^{(f)}$

(a) Department of Endodontics, Dental School, Universidade Cruzeiro do Sul - Unicsul, São Paulo, SP, Brazil.

(b) Department of Endodontics, Dental School, Univ. Estad. Paulista - UNESP, São José dos Campos, SP, Brazil.

(c) Department of Biochemistry, Dental School, Univ. Estad. Paulista - UNESP, São José dos Campos, SP, Brazil.

(d) Ultradent Products, Inc., South Jordan, UT, USA.

(e) Department of Endodontics, Dental School, Univ. Estad. Paulista - UNESP, Araraquara, SP, Brazil.

(f) Department of Endodontics, Dental School, Universidade de São Paulo - USP, São Paulo, SP, Brazil.

Declaration of Interests: The authors certify that they have no commercial or associative interest that represents a conflict of interest in connection with the manuscript.

Corresponding Author:

Cacio Moura-Netto

E-mail:caciomn@usp.br

Received for publication on Feb 03, 2012 Accepted for publication on Apr 25, 2012
Abstract: Dentin wall structural changes caused by 810 -nm-diode laser irradiation can influence the sealing ability of endodontic sealers. The objective of this study was to evaluate the apical leakage of AH Plus and RealSeal resin-based sealers with and without prior diode laser irradiation. Fifty-two single-rooted mandibular premolars were prepared and divided into 4 groups, according to the endodontic sealer used and the use or non-use of laser irradiation. The protocol for laser irradiation was $2.5 \mathrm{~W}$, continuous wave in scanning mode, with 4 exposures per tooth. After sample preparation, apical leakage of $50 \%$ ammoniacal silver nitrate impregnation was analyzed. When the teeth were not exposed to irradiation, the RealSeal sealer achieved the highest scores, showing the least leakage, with significant differences at the 5\% level (Kruskal-Wallis test, $\mathrm{p}=0.0004)$, compared with AH Plus. When the teeth were exposed to the 810-nm-diode laser irradiation, the sealing ability of AH Plus sealer was improved $(\mathrm{p}=0282)$. In the RealSeal groups, the intracanal laser irradiation did not interfere with the leakage index, showing similar results in the GRS and GRSd groups ( $\mathrm{p}=0.1009)$.

Descriptors: Dental Pulp Cavity; Root Canal Filling Materials; Laser Therapy; Root Canal Therapy; Endodontics.

\section{Introduction}

The complete seal of a canal system is extremely important for achieving success in endodontic therapy. Many products have been tested for complete disinfection and sealing of the root canal system. ${ }^{1,2}$ The use of laser devices, such as the Nd:YAG and diode lasers, has been shown to be a great asset for root canal treatment in endodontics. ${ }^{3-6}$ Diode laser irradiation has been proposed for endodontic therapy and has been shown to increase the level of disinfection, as well as produce morphological changes that occlude dentin tubules, improving the seal of the root canal system. ${ }^{3,4,6-12}$ There are several studies about materials and root canal filling techniques that aim to achieve apical sealing of teeth. ${ }^{13-17}$ Currently, studies with Epiphany (Pentron Clinical, Orange, USA) and RealSeal (Sybron Endo, Redmond, USA), both methacrylate-based resin sealers, have shown promising results in comparison with other base sealers.,18,19 However, it is not well-known if the combined association of intracanal 
laser irradiation and methacrylate-based resin sealers, which separately improve an endodontic seal, could enhance endodontic treatment.

Thus, the objective of this study was to evaluate the apical leakage of AH Plus and RealSeal resinbased sealers with and without prior diode laser irradiation.

\section{Methodology}

This study was approved by the Ethics Committee at the Dentistry College of the University of São Paulo (Protocol 92/2008). Fifty-two single-rooted mandibular premolars, stored in $1 \%$ thymol solution, were selected from a tooth bank from the same institution. After coronal access, a size $15 \mathrm{~K}$-file (Dentsply Maillefer, Ballaigues, Switzerland) was inserted into the root canal until the tip was visible at the apical foramen, and a working length $1.0 \mathrm{~mm}$ short from the apical foramen was established. All teeth were prepared by the "crown down" technique, utilizing .04 taper K3 rotary files (Sybron Endo, Redmond, USA) up to size 40 with $2.5 \%$ sodium hypochlorite. Once the preparation was complete, each root canal was flushed with $10 \mathrm{~mL}$ of $2.5 \% \mathrm{NaOCl}$ followed by $10 \mathrm{~mL}$ of a $17 \%$ EDTA-T solution and a final rinse of $20 \mathrm{~mL}$ of sterile water to remove the other irrigants. The samples were then randomly divided into four experimental groups $(n=12)$ according to the endodontic sealer used and whether canals were lased or non-lased.

\section{Diode laser irradiation}

For the irradiated groups, an 810-nm-wavelength-diode laser device (Twilite with laserSmile, Biolase Technology Inc., Irvine, USA), set at $2.5 \mathrm{~W}$ continuous wave in scanning mode, was used. ${ }^{6-7}$ With the root canal moistened with sterile water, the optical fiber tip $(0.3 \mathrm{~mm}$ in diameter) was inserted into the root canal $1 \mathrm{~mm}$ short of the working length without activating the laser. Once the tip was in position, the laser was initiated, and the fiber worked in contact mode, moving coronally in circular movements over the root canal wall at a speed of $2 \mathrm{~mm} / \mathrm{s}$ (calculated according to the working length of each tooth, based on a time of $0.5 \mathrm{~s}$ of irradiation per millimeter). This process was repeated 4 times with 20-second intervals between exposures. Assuming that the mean root length of the sample was $15 \mathrm{~mm}$, it could be calculated that the total irradiation time for each tooth was approximately $28 \mathrm{~s}$.

\section{SEM analysis}

Two lased and two non-lased roots were split (by fracture) in the buccal-lingual direction, and the sections were dehydrated by a series of graded ethanol solutions, and then coated with a gold layer after drying. The selected dentinal surface of the apical third, $3 \mathrm{~mm}$ from the apex and equidistant from lateral walls, was observed by scanning electron microscopy (SEM) (XL30, Philips, Eindhoven, Netherlands) at a working distance of $10 \mathrm{~mm}$ and beam acceleration of $10 \mathrm{kV}$. The surface topography of non-lased roots and the extent of morphologic changes caused by laser irradiation were analyzed in comparison with those in the non-irradiated group.

\section{Root canal filling}

The root canals of all four groups were filled by the lateral condensation technique. ${ }^{12}$ The canals of the AH Plus sealer (Dentsply, Konstanz, Germany) groups were then dried with suction (0.014" Capillary tips, Ultradent Products, Salt Lake City, USA), and paper cones (Dentsply Maillefer) and a taper (0.4 \#40 size gutta-percha cone; Dentsply Maillefer) were fitted to the working length. AH Plus sealer was mixed according to the manufacturer's instructions and placed in the canal with the master cone, which was carefully seated to working length. Lateral condensation was then completed by the placement of accessory gutta-percha cones with endodontic finger-spreaders (Dentsply Maillefer) until the spreader could not enter more than $3 \mathrm{~mm}$.

The RealSeal sealer (SybronEndo) groups were dried with suction and 1 or 2 paper cones, to maintain moisture in the dentin walls. Following the manufacturer's instructions, RealSeal sealer and taper .04 \#40 Resilon cones (Resilon Research, LLC, Madison, USA) were placed by a lateral condensation technique. The excess of cone and sealer from each of the groups was removed with a hot plugger and vertically condensed by means of a cold plugger. The coronal openings of all samples were sealed with 
glass-ionomer cement and stored in $37^{\circ} \mathrm{C}$ and $100 \%$ humidity for $48 \mathrm{~h}$. All teeth were radiographed in bucco-lingual and mesial-distal positions for the assessment of filling quality. The teeth were then externally coated with two layers of ethyl cyanoacrylate (Superbonder, Loctite, São Paulo, Brazil), except for the last $2 \mathrm{~mm}$ of the apical third. The specimens were immersed and kept in 50\% silver nitrate solution for $24 \mathrm{~h}$, followed by a thorough rinse in running water. Each specimen was then embedded in acrylic blocks and split into two sections, longitudinally. Next, the hemi-sections were rinsed and placed in photodeveloper solution (Kodak Eastman, Rochester, USA) for $8 \mathrm{~h}$ to reduce the silver ions to metallic silver. ${ }^{20}$ The samples were then scanned with a high-opticalresolution scanner (24000 dpi - CX7300, Epson, São Paulo, Brazil), and the impregnation on each side of the sample was blindly measured (mean value), with Image J Software (NIH, Bethesda, USA). The leakage measures were converted to scores ( 1 to 6 ; see Table 1) and subjected to the Kruskal-Wallis test for nonparametric data to determine whether there were statistically significant differences $(\alpha=0.05)$ between and among the groups. If statistically significant differences were present, pairs of groups were compared by the Student-Newman-Keuls test.

\section{ESEM analysis of resin tag penetration}

Assessment of the penetration of the sealers into dentinal tubules (presence of tags) was used to perform the qualitative analysis; two sections of the roots of each group were randomly chosen and prepared for observation. The root surfaces were conditioned with a $37 \%$ phosphoric acid solution for $30 \mathrm{~s}$, followed by irrigation with distilled water, immersion in $2.5 \%$ sodium hypochlorite for $10 \mathrm{~min}$, and a final flush with distilled water. ${ }^{21}$ Non-dehydrated and uncoated specimens were fixed for platform-specific analysis in an environmental scanning electron microscope (ESEM) (Quanta 600 FEG, FEI Company, Eindhoven, Netherlands). To standardize the surfaces analyzed, we chose the ESEM images of the apical root third at a distance of $4 \mathrm{~mm}$ from the edge of the cone, in mesial and distal interfaces between the dentin wall and filling material (magnification, 1500×).

\section{Results}

SEM analysis showed that the non-lased surfaces presented clean dentin walls with open dentin tubules. The canals irradiated with the diode laser showed melting and re-solidification of the dentin surface and partially occluded dentin tubules (Figure 1).

Table 1 shows a frequency distribution of the leakage scores of the four groups, with the RealSeal groups exhibiting lower leakage scores than the AH Plus groups. In the non-lased groups, RealSeal sealer showed significantly lower leakage rates than AH Plus ( $\mathrm{p}=0.0004)$. When lased and unlased groups were compared, it was observed that the 810-nm-diode laser improved the sealing ability of AH Plus sealer, with a statistically significant difference $(\mathrm{p}=0.0282)$. In the RealSeal groups, intracanal laser irradiation did not interfere with the leakage index, and similar results were observed in the GRS and GRSd groups ( $\mathrm{p}=0.1009)$.

Figure 2 shows ESEM images of resin tag penetration in the experimental groups. It can be observed that the penetration of sealer was similar among the experimental groups independent of the laser application $(\mathrm{p}<0.05)$. The resin tag penetra-

\begin{tabular}{|c|c|c|c|c|c|c|c|c|c|c|}
\hline \multirow{6}{*}{$\begin{array}{r}\text { Table } 1 \text { - Distribution of } \\
\text { leakage scores, median and } \\
\text { means } \pm \text { standard deviations ( } \mathrm{mm} \text { ) } \\
\text { of the experimental groups. }\end{array}$} & \multirow{2}{*}{ Group } & \multicolumn{6}{|c|}{ Scores* } & \multirow[b]{2}{*}{ median } & \multirow[b]{2}{*}{ mean $\pm s d$} & \multirow{2}{*}{$\begin{array}{l}\text { Kruskal- } \\
\text { Wallis }\end{array}$} \\
\hline & & 1 & 2 & 3 & 4 & 5 & 6 & & & \\
\hline & GAH (AH Plus) & - & - & 2 & 4 & 4 & 2 & 4.5 & $2.04 \pm 0.46$ & A \\
\hline & GAHd (AH Plus + diode laser) & - & - & 6 & 5 & 1 & - & 3.5 & $1.57 \pm 0.28$ & B \\
\hline & GRS (RealSeal) & - & 4 & 7 & 1 & - & - & 3 & $1.20 \pm 0.26$ & C \\
\hline & GRSd (RealSeal + diode laser) & - & 1 & 8 & 3 & - & - & 3 & $1.34 \pm 0.24$ & $B, C$ \\
\hline
\end{tabular}



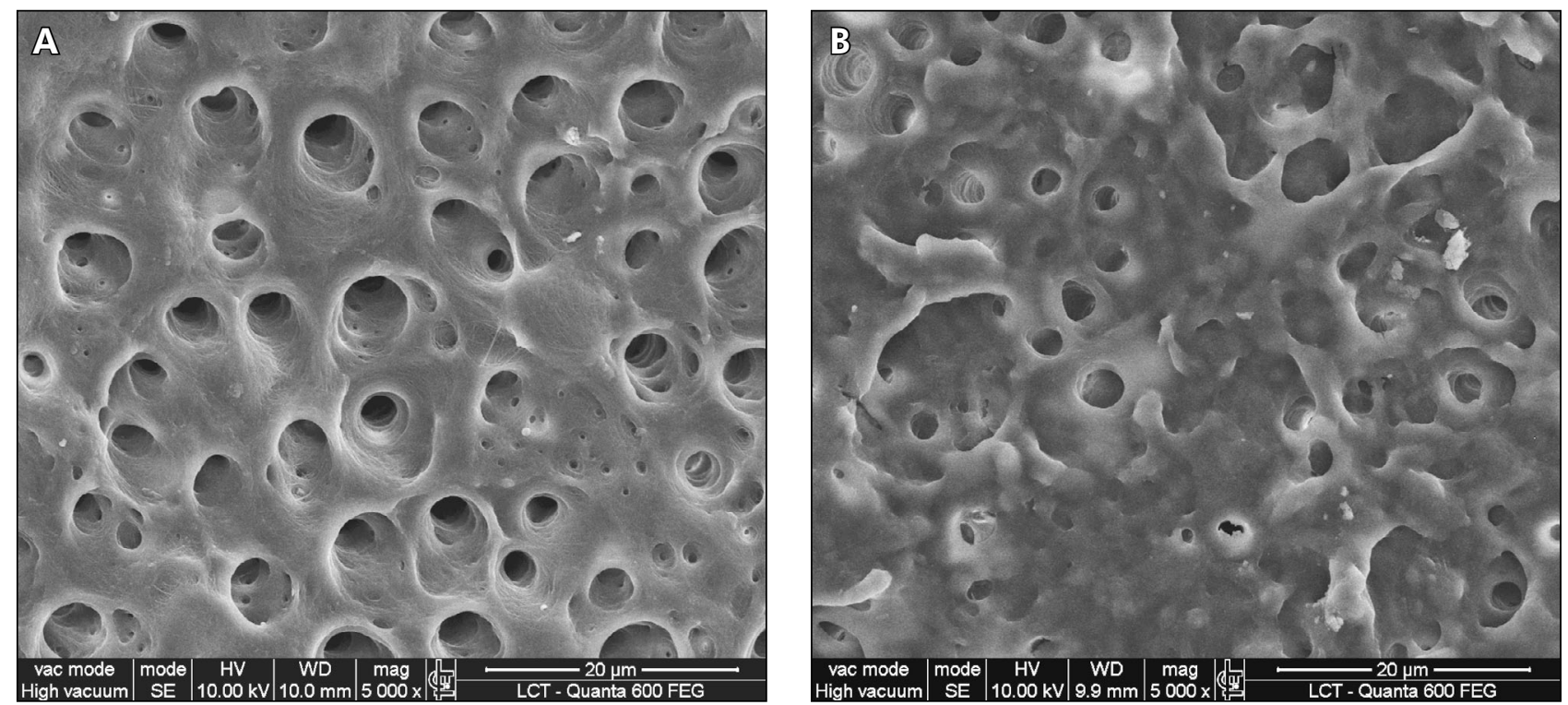

Figure 1 - SEM images of the dentin surface without laser irradiation (A), showing a clean wall free of smear layer and open dentin tubules; after diode laser irradiation (B), melting of the dentin surface can be noted, along with a few dentin tubules, most of them occluded.

tion of RealSeal sealer was apparently deeper than that of AH Plus. In a comparison of the RealSeal groups, a higher concentration of resin tags was noted in the non-lased group.

\section{Discussion}

The perfect apical seal of a root canal system is the goal of successful endodontic treatment. ${ }^{2}$ After endodontic instrumentation, but prior to filling of the root canal, the treatment of the root canal wall may influence the marginal sealing of the filling, as has been demonstrated in studies with chemical substances, such as EDTA,,$^{1,2,13-15}$ or with laser irradiation. ${ }^{4,11-12,22}$ The high intensity of the diode laser has notable effects on intracanal irradiation, including bacterial reduction ${ }^{8,10}$ and melting and resolidification of dentin walls, with partial occlusion of dentinal tubules. ${ }^{6,9}$ Moura-Netto et al. ${ }^{6}$ showed similar results when comparing Nd:YAG and diode laser irradiation with regard to morphologic changes and debris removal on the intracanal dentin surface. The diode laser melted the dentin, creating a more uniform circular pattern than the Nd:YAG laser, and apparently occluded several dentin tubules. In that study, the authors did not find statistical differences when the effects of these two laser de- vices were compared. Therefore, these effects could increase the sealing success of the endodontic treatment. ${ }^{4}$ In addition, research completed over the last few years, evaluating apical leakage of new methacrylate resin-based endodontic sealers, has shown good results. ${ }^{2,18}$

In the evaluation of different endodontic techniques, dye is commonly used for in vitro studies as an indicator of apical leakage, which is illustrated by the dye penetration between the canal walls and the obturating materials. ${ }^{18,22,23}$ In this study, 50\% silver nitrate impregnation was used to measure apical leakage. This method is commonly used to assess micro- and nanoleakage of composite fillings, by tracing gaps and spaces between the composite and dentin walls. ${ }^{17,20,22}$ As with any other method, dye or tracing tests do not represent or simulate the exact behavior of these sealers in clinical conditions. However, since there is no single, universally accepted model of leakage testing in endodontics, this leakage test can be used to compare the sealing abilities of the tested materials. ${ }^{23}$

Several studies concerning root canal sealers classify AH Plus sealer as the product with the lowest apical marginal dye index..$^{4,14-16,24,25}$ However, as found in this and other studies (e.g., Onay et al.), ${ }^{26}$ 

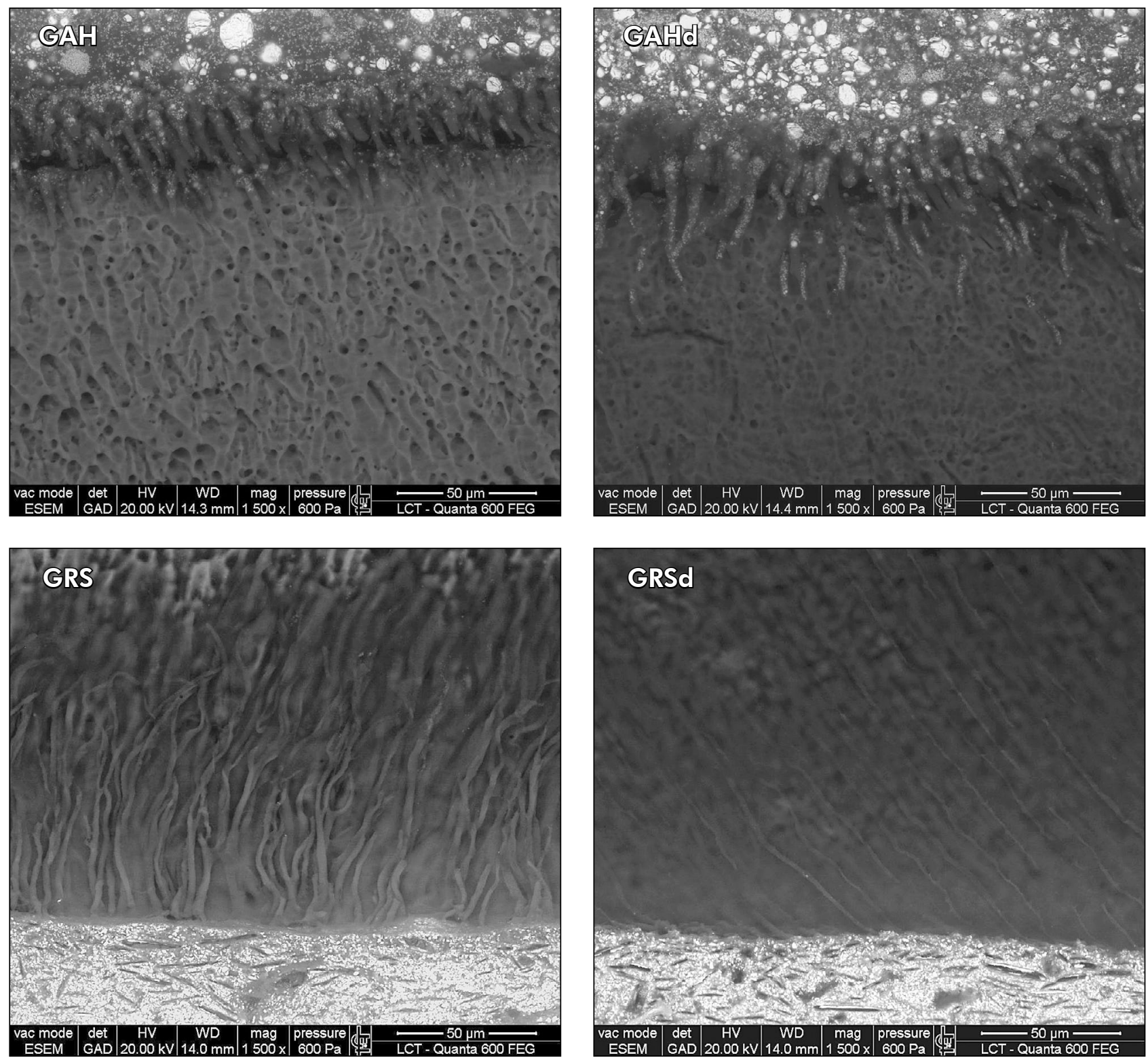

Figure 2 - ESEM images of resin tag penetration in the experimental groups. GAH: AH Plus; GAHd: AH Plus + diode laser; GRS: RealSeal; GRSd: RealSeal + diode laser. Resin tags appeared longer in the RealSeal groups than in the AH Plus groups.

better microleakage scores, denoting better apical sealing, were achieved with the use of methacrylatebased sealers in comparison with AH Plus..$^{27,28}$

The present study compares a hydrophobic epoxy resin sealer with a hydrophilic methacrylate resin-based sealer, with the additional variable of irradiation by the 810 -nm-diode laser. Differences in the mean values and scores of apical leakage between the tested cements are evident. It could be noted that the RealSeal groups (GRS and GRSd) promoted better apical sealing, followed by AH Plus + laser (GAHd) and AH Plus (GAH).

These findings should be a consideration for general practitioners and endodontists who associate new technologies and materials with increased endodontic treatment success. In our results, the leakage index decreased in the GAHd group, showing a tendency of compatibility between a diode laser and epoxy resin-based sealer. However, laser intracanal irradiation did not seem to be useful in promoting 
better apical sealing on methacrylate resin-based sealers.

Also, in this study, the penetration of sealers into the dentinal tubules was observed by ESEM. It can be observed that the penetration of sealers was similar among the experimental groups, independent of the laser application. However, it seems that Real Seal (GRS and GRSd) showed a deeper penetration than the other sealers. This could be explained by

\section{References}

1. Shipper G, Ørstavik D, Teixeira FB, Trope M. An evaluation of microbial leakage in roots filled with a thermoplastic synthetic polymer-based root canal filling material (Resilon). J Endod. 2004 May;30(5):342-7.

2. Moura-Netto C, Pinto T, Davidowicz H, de Moura AA. Apical leakage of three resin-based endodontic sealers after 810-nm-diode laser irradiation. Photomed Laser Surg. 2009 Dec;27(6):891-4.

3. Kimura Y, Wilder-Smith P, Matsumoto K. Lasers in endodontics: a review. Int Endod J. 2000 May;33(3):173-85.

4. Moura-Netto C, Carvalho CF, Moura AAM, Davidowicz $\mathrm{H}$, Antoniazzi JH. Influence of Nd:YAG and diode laser irradiation on apical sealing when associated with $\mathrm{AH}$ plus and EndoREZ endodontic cements. Photomed Laser Surg. 2007 Oct;25(5):413-7.

5. Folwaczny M, Mehl A, Jordan C, Hickel R. Antibacterial effects of pulsed Nd:YAG laser radiation at different energy settings in root canals. J Endod. 2002 Jan;28(1):24-9.

6. Moura-Netto C, Moura AAM, Davidowicz H, Aun CE, Antonio MPS. Morphologic changes and removal of debris on apical dentin surface after Nd:YAG laser and diode laser irradiation. Photomed Laser Surg. 2008 Jun;26(3):263-6.

7. da Costa Ribeiro A, Nogueira GE, Antoniazzi JH, Moritz A, Zezell DM. Effects of diode laser $(810 \mathrm{~nm})$ irradiation on root canal walls: thermographic and morphological studies. J Endod. 2007 Mar;33(3):252-5.

8. Gutknecht N, Van Gogswaardt D, Conrads G, Apel C, Schubert C, Lampert F. Diode laser radiation and its bactericidal effect in root canal wall dentin. J Clin Laser Med Surg. 2000 Apr;18(2):57-60.

9. Kreisler M, Al Haj H, Daublander M, Gotz H, Duschner H, Willershausen B, et al. Effect of diode laser irradiation on root surfaces in vitro. J Clin Laser Med Surg. 2002 Apr;20(2):63-9.

10. Moritz A, Gutknecht N, Schoop U, Goharkhay K, Doertbudak $\mathrm{O}$, Sperr W. Irradiation of infected root canals diode laser in vivo: results of microbiological examinations. Lasers Surg Med. 1997 Sep;21(3):221-6.

11. Wang X, Sun Y, Kimura Y, Kinoshita J, Ishizaki NT, Matsumoto K. Effects of diode laser irradiation on smear layer the hydrophilicity of this sealer, which improves its penetration into dentinal tubules.

\section{Conclusion}

According to our results, Real Seal root canal sealer showed better apical leakage results than $\mathrm{AH}$ Plus. Previous diode laser irradiation improved the sealing ability of AH Plus cement, but did not interfere with Real Seal obturation.

removal from root canal walls and apical leakage after obturation. Photomed Laser Surg. 2005 Dec;23(6):575-81.

12. Moura-Netto C, Guglielmi CA, Mello-Moura AC, Palo RM, Raggio DP, Caldeira CL. Nd:YAG laser irradiation effect on apical intracanal dentin - a microleakage and SEM evaluation. Braz Dent J. 2011 Oct;22(5):377-81.

13. Tanomaru-Filho M, Sant'anna-Junior A, Bosso R, GuerreiroTanomaru JM. Effectiveness of gutta-percha and Resilon in filling lateral root canals using the Obtura II system. Braz Oral Res. 2011 May-Jun;25(3):205-9.

14. Zmener O, Spielberg C, Lamberghini F, Rucci M. Sealing properties of a new epoxy resin-based root canal sealer. Int Endod J. 1997 May;30(5):332-4.

15. Kqiku L, Städtler P, Gruber HJ, Baraba A, Anic I, Miletic I. Active versus passive microleakage of Resilon/Epiphany and gutta-percha/AH Plus. Aust Endod J. 2011 Dec;37(3):141-6.

16. Kardon BP, Kuttler S, Hardigan P, Dorne SO. An in vitro evaluation of the sealing ability of a new root-canal obturation system. J Endod. 2003 Oct;29(10):658-61.

17. Vogt BF, Xavier CB, Demarco FF, Padilha MS. Dentin penetrability evaluation of three different dyes in root-end cavities filled with mineral trioxide aggregate (MTA). Braz Oral Res. 2006 Apr-Jun;20(2):132-6.

18. Verissimo DM, do Vale MS, Monteiro AJ. Comparison of apical leakage between canals filled with gutta-percha/AH-Plus and the Resilon/Epiphany System, when submitted to two filling techniques. J Endod. 2007 Mar;33(3):291-4.

19. Teixeira FB, Teixeira EC, Thompson J, Leinfelder KF, Trope M. Dentinal bonding reaches the root canal system. J Esthet Restor Dent. 2004 Jun;16(6):348-54; discussion 354.

20. Tay FR, Pashley DH, Yoshiyama M. Two modes of nanoleakage expression in single-step adhesives. J Dent Res. 2002 Jul;81(7):472-6.

21. Tay FR, Loushine RJ, Monticelli F, Weller RN, Breschi L, Ferrari $\mathrm{M}$, et al. Effectiveness of resin-coated gutta-percha cones and a dual-cured, hydrophilic methacrylate resin-based sealer in obturating root canals. J Endod. 2005 Sep;31(9):659-64.

22. Tamse A, Katz A, Kablan F. Comparison of apical leakage shown by four different dyes with two evaluating methods. Int Endod J. 1998 Sep;31(5):333-7. 
23. Wu M-K, Wesselink PR. Endodontic leakage studies reconsidered, Part 1. Methodology, application and relevance. Int Endod J. 1993 Jan;26(1):37-43.

24. da Silva Neto UX, de Moraes IG, Westphalen VP, Menezes R, Carneiro E, Fariniuk LF. Leakage of 4 resin-based root-canal sealers used with a single-cone technique. Oral Surg Oral Med Oral Pathol Oral Radiol Endod. 2007 Aug;104(2):e53-7.

25. Haïkel Y, Wittenmeyer W, Bateman G, Bentaleb A, Alleman C. A new method for the quantitative analysis of endodontic microleakage. J Endod. 1999 Mar;25(3):172-7.
26. Onay EO, Ungor M, Orucoglu H. An in vitro evaluation of the apical sealing ability of a new resin-based root canal obturation system. J Endod. 2006 Oct;32(10):976-8.

27. Bodrumlu, E, Tunga, U. Apical leakage of Resilon obturation material. J Contemp Dent Pract. 2006 Sep 1;7(4):45-52.

28. Stratton RK, Apicella MJ, Mines P. A fluid filtration comparison of gutta-percha versus Resilon, a new soft resin endodontic obturation system. J Endod. 2006 Jul;32(7):642-5. 